\title{
Risk of dementia after stroke in a hospitalized cohort: Results of a longitudinal study
}

\author{
T.K. Tatemichi, MD; M. Paik, PhD; E. Bagiella, MS; D.W. Desmond, PhD; Y. Stern, PhD; M. Sano, PhD; \\ W.A. Hauser, MD; and R. Mayeux, MD
}

\begin{abstract}
Article abstract-Stroke is considered the second most common cause of dementia, but the magnitude of the risk posed by stroke has not been fully clarified. The aim of this study was to determine the long-term risk of developing dementia after stroke onset in a hospitalized cohort. We prospectively examined 185 nondemented patients aged $\geq 60$ years hospitalized with ischemic stroke and 241 age-matched nondemented controls without stroke from the same community using neurologic, neuropsychological, and functional assessments given annually. Using criteria modified from the DSM-III-R, we diagnosed incident dementia based on the annual examination findings. We used life-table methods to estimate incidence in the two groups, Kaplan-Meier analysis to determine the proportion surviving without dementia, and Cox proportional-hazards analysis to compute the relative risk (RR) of dementia after 1 to 4 years of follow-up. The incidence of dementia was 8.4 per 100 person-years in the stroke group and 1.3 per 100 person-years in the control group. After 52 months of follow-up, the cumulative proportion $( \pm \mathrm{SE})$ surviving without dementia was 66.3 $\pm 5.5 \%$ for stroke and $90.3 \pm 4.3 \%$ for control subjects. The RR of dementia associated with stroke compared with controls was 5.5 (95\% CI, 2.5 to 11.1) after adjusting for demographic factors. Older age at stroke onset and fewer years of education were significant covariates, but sex and race were not. A low score on the Mini-Mental State Examination at baseline was a significant predictor when added to this model. We conclude that ischemic stroke in elderly persons increases the long-term risk of developing dementia by approximately five-fold compared with those without stroke. Age, education, and baseline intellectual function contribute independently to that risk.
\end{abstract}

NEUROLOGY 1994;44:1885-1891

Dementia associated with cerebrovascular disease (CVD) is commonly recognized as an immediate consequence of stroke, with intellectual decline measurable soon after one or more ischemic events. ${ }^{1}$ In a previous study, we found dementia in approximately one of four patients examined 3 months after hospitalization for acute ischemic stroke. $^{2}$ Compared with a stroke-free sample, the odds of dementia associated with stroke were at least nine times greater. Although our initial study demonstrated that stroke increased the risk of dementia early after its onset, it did not address whether stroke can lead to delayed intellectual consequences measurable 1 or more years after a single event.

To extend our cross-sectional observations, we conducted a prospective study to determine the incidence of dementia among stroke subjects who were not demented at 3 months. Studies of inci- dence generally provide a more precise estimate of the risk of a disorder. ${ }^{3}$ We hypothesized that a "delayed" dementia may follow a well-defined stroke event, and that the incidence of dementia would be more frequent in these patients than among a stroke-free reference group.

Methods. Subjects and setting. Patients were recruited among those aged $\geq 60$ years who were consecutively admitted to Columbia-Presbyterian Medical Center between September 1, 1988, and December 31, 1990, within 30 days of acute ischemic stroke. ${ }^{2}$ Patients with a prior stroke were included, but those with severe aphasia were excluded. The diagnosis of stroke was supported by CT (relevant infarct or negative) obtained as part of the clinical evaluation for stroke. During hospitalization, patients underwent structured medical and neurologic examinations, which included functional ratings using the Barthel Index ${ }^{4}$ and Blessed Functional Activity Scale. ${ }^{5}$

The control group was composed of 241 community-re-

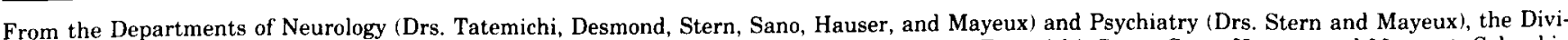

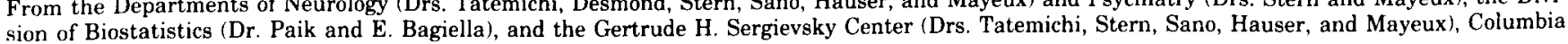
University, College of Physicians and Surgeons, New York, NY.

Supported in part by Grants R01-NS26179 and P01-AG07232 from the National Institutes of Health.

Presented in part at the 45th annual meeting of the American Academy of Neurology, New York, NY, April 1993.

Received January 24, 1994. Accepted in final form March 16, 1994.

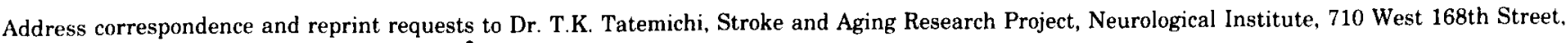
New York, NY 10032 . 
siding volunteers aged $\geq 60$ years without historical or clinical examination evidence of stroke or dementia who were recruited during the same time period. The group was composed of spouses of stroke subjects, a probability sample from Medicare files, and neighborhood volunteers who were matched as a group by age to the stroke sample. Details of our recruitment procedures were described in a report on methods and baseline findings. ${ }^{2}$ Informed consent was obtained from subjects or their family members according to the procedures of the Institutional Review Board of Columbia University.

Diagnostic methods. Approximately 3 months after stroke onset, patients received a battery of neuropsychological tests, ${ }^{6,7}$ the Hamilton Depression Rating Scale (HDRS) ${ }^{8}$ and the Mini-Mental State Examination (MMSE). ${ }^{9}$ Neurologic and functional examinations were repeated. Controls received the same clinical assessments. Based on combined clinical information reviewed at a consensus conference, dementia was diagnosed using criteria modified from the DSM-III-R. ${ }^{10}$ We required the presence of memory impairment as well as deficits in two other cognitive domains (ie, orientation, abstract reasoning, language, visuospatial function) combined with functional impairment considered unrelated to physical deficits. ${ }^{2}$ Impairment within each cognitive domain was defined by performance on neuropsychological tests falling below pre-defined cutoff scores that were developed in prior studies. ${ }^{6}$ We have reported ${ }^{7}$ norms for this battery in our stroke and control subjects and consider the cutoffs to be conservative. Agreement on the diagnosis of dementia was excellent, with a kappa of 0.96 based on independent judgments in a sample of 63 subjects. Among the 251 stroke subjects examined at baseline, 66 were judged to have dementia. Their clinical features have been described previously. ${ }^{11}$ The remaining 185 nondemented patients comprised the inception stroke cohort, and the 241 nondemented, stroke-free, age-matched subjects served as the control cohort for this incidence study.

Follow-up and outcomes. Stroke and control subjects were examined annually by re-administering the clinical assessments. Annual follow-up was based on the date of stroke onset for stroke subjects and the initial enrollment date for controls. For the list-learning task in the neuropsychological battery, different word lists of equivalent difficulty were used to minimize practice effects; otherwise, the test battery remained identical. Interval medical history focused on the occurrence of new ischemic events, the development of new major comorbidity or hospitalizations, and the use of medications that might affect cognitive function. Follow-up CT of the brain was available in a limited number of subjects; whenever possible, the results were reviewed for subjects who had recurrent stroke, incident dementia, or both. To maximize follow-up rates, we made visits to the subjects' homes or health care facilities if they were unwilling or unable to visit the medical center, and did not consider them as "refusals" for that interval until at least four attempts had been made to contact and examine them. When in-person examinations were not possible, we obtained information by telephone to ascertain vital status and the occurrence of major clinical events.

Using the same diagnostic procedure described above, incident dementia was diagnosed in stroke and control subjects. We did not require a specific degree of decrement in performance for the diagnosis of incident dementia; only performance below the cutoff scores combined with functional impairment was required. These proce- dures were intended to maximize consistency and reliability in diagnosis over time. ${ }^{12}$ When dementia was diagnosed, the date of the annual clinical visit was taken as the time of onset of new dementia. A Clinical Dementia Rating (CDR) ${ }^{13}$ was assigned based on clinical, functional, and neuropsychological information. Each subject was followed until death or June 30,1993 , the end of the incidence study period.

Data analysis and statistics. Newly demented subjects were enumerated in the stroke and control cohorts, and the crude incidence rate (IR) of dementia was calculated using life-table methods. The clinical characteristics of the stroke patients with incident dementia were summarized. Kaplan-Meier survival analysis ${ }^{14}$ was used to determine the proportion surviving free of dementia in our stroke and control cohorts overall. To compute the relative risk (RR) of dementia associated with stroke compared with controls, we used Cox proportional-hazards analysis. ${ }^{15}$ Although the stroke and control groups were closely matched by age, there were significant differences by education, race, and sex. ${ }^{2}$ To adjust for potential confounding by these demographic factors, the following variables were included in the Cox model: age $(80+$ and 70 to 79 years versus 60 to 69 years), education ( $\leq 8$ and 9 to 12 years versus $13+$ years), race (nonwhite versus white), and sex (male versus female), in addition to subject status (stroke versus control). We used trichotomous categories for age and education (even though their effects were linear) to facilitate interpretation of the RR by substrata. Using this multivariate procedure, an adjusted RR for stroke compared with controls was estimated. To adjust for potential recruitment biases, we developed a second model, confining the Cox analysis to the sample of subjects (both cases and controls) residing in the Washington Heights-Inwood community of northern Manhattan defined by five zip code zones (10032, 10033, $10034,10039,10040$ ). This population-based approach has been suggested as one method to control for the possibility of differential referral in hospital-based samples. ${ }^{16}$ Finally, to adjust for the severity of cognitive impairment, we added the MMSE score from the baseline examination (ie, 3 months after stroke or the initial examination for controls) to the first model.

Results. Unadjusted IRs. Among 185 stroke subjects, $154(83.2 \%)$ completed at least one visit after the baseline, $132(71.4 \%)$ completed two visits, 73 (39.5\%) completed three visits, and $26(14.1 \%)$ completed at least four visits. Deaths occurred in 34 (18.4\%). Excluding deaths occurring before each planned follow-up interval, the proportion of patients examined among those at risk for incident dementia was $82.9 \%$ at year $1,91.2 \%$ at year 2 , $89.7 \%$ at year 3 , and $91.3 \%$ at year 4 . During 427 person-years of follow-up in the stroke group, a total of 36 subjects were found to have new dementia, resulting in an IR of 8.4 cases per 100 personyears.

Among 241 control subjects, 203 (84.2\%) completed at least one visit after the baseline, 188 $(78.0 \%)$ completed two visits, 91 (37.8\%) completed three visits, and $35(14.5 \%)$ completed at least four visits. Deaths occurred in eight $(3.3 \%)$. Excluding deaths occurring before each planned follow-up interval, the proportion of controls examined among 
those at risk for incident dementia was $83.5 \%$ at year $1,93.0 \%$ at year $2,86.3 \%$ at year 3 , and $97.1 \%$ at year 4 . During 614.5 person-years of follow-up in the control group, a total of eight subjects were found to have new dementia, resulting in an IR of 1.3 cases per 100 person-years. All eight of the newly demented controls were judged clinically to have Alzheimer's disease (AD), and none had suffered a clinically evident stroke during the followup period.

Clinical features of stroke subjects with incident dementia. Characteristics of the 36 stroke subjects with incident dementia are summarized in table 1. All but six (16.7\%) of the 36 were judged to have mild dementia with a CDR of 1 . Although MMSE scores are shown at each examination interval, it should be emphasized that the diagnosis of incident dementia was based on findings from neuropsychological and functional examinations. Using the MMSE as a guide to the course of cognitive function over time, however, few patients showed a smoothly progressive deterioration, as seen typically in AD. Rather, fluctuations were the rule, and, in some, a steep decline occurred in association with intercurrent illness.

A clinical stroke event occurred in eight $(22.2 \%)$ of the 36 incident cases (patients $3,9,11,21,22$, 24,29 , and 35 ). Brain imaging revealed new infarction in these eight patients, and incident dementia was judged related to the new stroke in seven of the eight. New medical comorbidity occurred during follow-up in eight $(22.2 \%)$ of the 36 incident cases (patients $1,2,4,12,13,21,31$, and 32 ), especially disorders or events that could have resulted in global cerebral ischemia or hypoxia (eg, seizures, syncope, congestive heart failure, and pneumonia). Only one subject (patient 28) had significant depression (HDRS score $=18$ ), which might have contributed to the new dementia. In reviewing specific changes in neuropsychological test scores over time, six (16.7\%) of the 36 cases (patients 5, 6, 10, $20,23,28$ ) had borderline scores at baseline, but did not meet our criteria for dementia at that point; subsequently, they qualified for a dementia diagnosis at the next visit. The remaining 12 subjects (33.3\%) showed significant neuropsychological worsening, but had no obvious new comorbidity, nor did any have new focal neurologic signs that might have indicated "silent" brain infarction.

The diagnosis of stroke-related dementia was confirmed by autopsy in one of our stroke patients with incident dementia (patient 10) who died 28 months after initial stroke onset and 18 months after the diagnosis of new dementia. Death occurred during a subsequent hospitalization for third-degree burns complicated by a recurrent stroke with stupor and left hemiplegia. Postmortem examination of the brain showed an extensive subacute hemorrhagic infarct involving the right frontal and parietal lobes consistent with both the recent event and the prior stroke, which involved a similar distribution on brain imaging. In addition, multiple small lacunar infarcts of the basal ganglia and internal capsule were evident. Multiple microscopic infarcts and broad areas of pallor and gliosis were seen in the centrum semiovale bilaterally. No neuritic plaques or neurofibrillary tangles were found in the hippocampus or frontal cortex.

Kaplan-Meier survival analysis. The cumulative proportion $( \pm \mathrm{SE})$ of subjects surviving free of dementia was $66.3 \pm 5.5 \%$ for stroke and $90.3 \pm 4.3 \%$ for control subjects after 52 months of follow-up (figure). Plots of the underlying hazard function supported the proportional-hazards model. By the log rank test, survival curves for the two groups were significantly different (chi squared $=29.42$, $p<0.001$ ).

Risk of dementia using the Cox model. In comparing the stroke and control cohorts, differences in demographic characteristics were evident, potentially confounding the estimation of dementia risk. Among 185 stroke subjects, the mean age $( \pm \mathrm{SD})$ was $70.3 \pm 7.7$, mean education was $10.5 \pm 4.7$, with $48.1 \%$ men and $36.8 \%$ white persons. Among 241 control subjects, the mean age was $70.5 \pm 6.5$, mean education was $12.4 \pm 4.5$, with $34.0 \%$ men and $53.1 \%$ white persons. We used a Cox model to estimate the RR of dementia adjusting for age, education, sex, and race (total $n=426$ ). The adjusted RR was 5.5 (table 2 , model A), with a $95 \%$ confidence interval (95\% CI) of 2.5 to 11.1. Significant covariates in this model included age and education. With the youngest subjects (60 to 69 years) serving as the reference group for age-specific comparisons, the RR by age in table 2 may be interpreted as follows: a subject (stroke or control) aged 80 years or older had a risk of dementia 5.7 times the risk for a subject aged 60 to 69 years. Similarly, with college-educated subjects (13+ years) serving as the reference group for education-specific comparisons, those with $\leq 8$ years of education had a three-fold increase in risk. The RRs for the stratified age and education subgroups suggested a trend towards increasing risk with advancing age and fewer years of education. Sex and race were not significant factors in this model.

In further refining this adjustment procedure, we confined the analysis to stroke and control subjects (total $n=180$ ) residing in the five zip code zones of the Washington Heights-Inwood community. The Cox model using this population-based sample resulted in an overall adjusted $R R$ of 7.2 (95\% CI, 2.6 to 20.3 ) as the risk of dementia after stroke (table 2, model B), similar to that for the overall sample. However, compared with model A, the age and education variables showed no risk trend by substrata.

Adding the baseline MMSE score as a continuous variable to the original model $(n=426)$, the estimated RR of dementia for stroke versus control subjects was 3.1 (95\% CI, 1.4 to 7.2$)$, while the effect of education was diminished, with a RR of 1.8 (95\% CI, 0.7 to 4.4 ) for $\leq 8$ years compared with $13+$ 


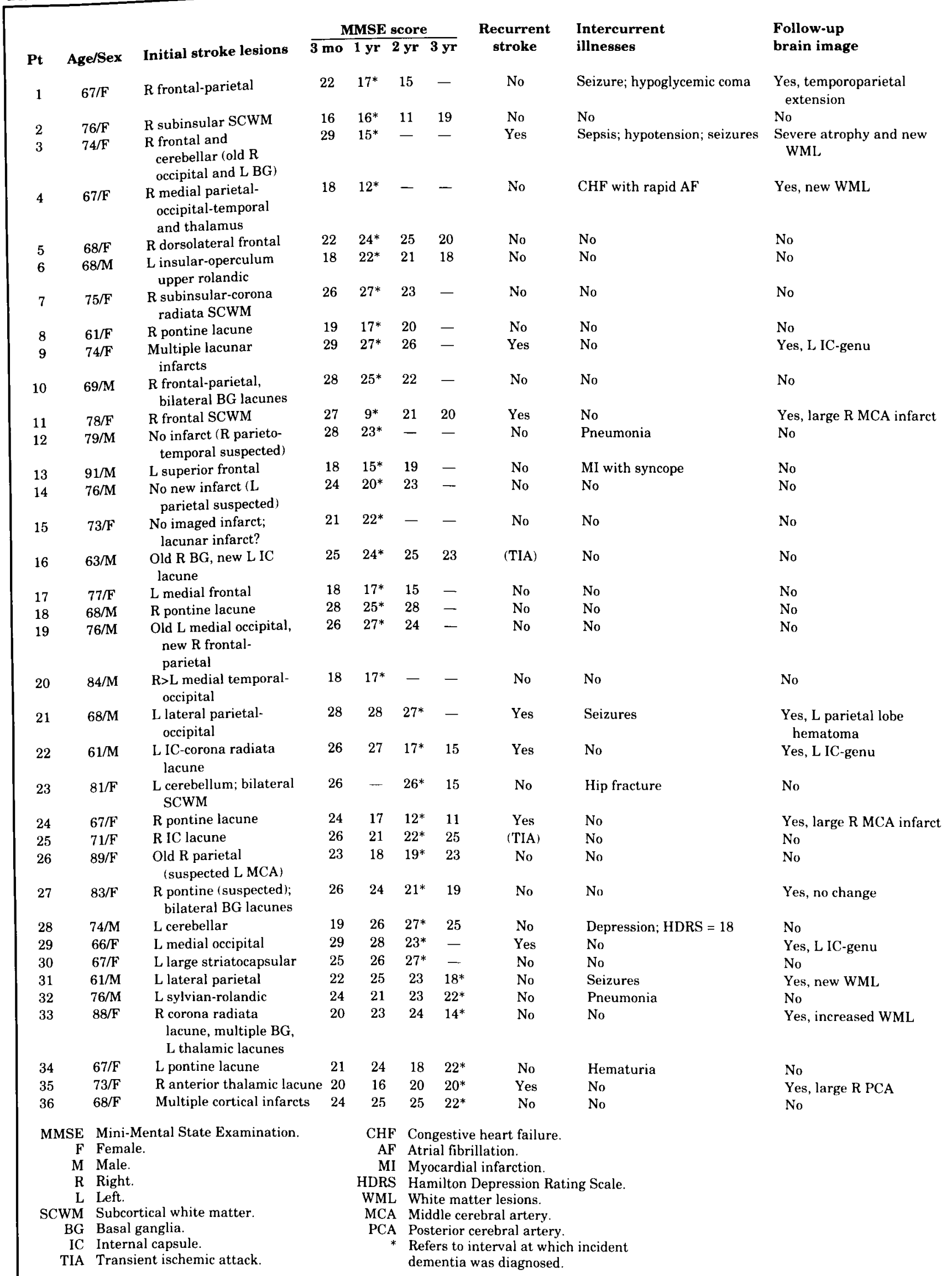




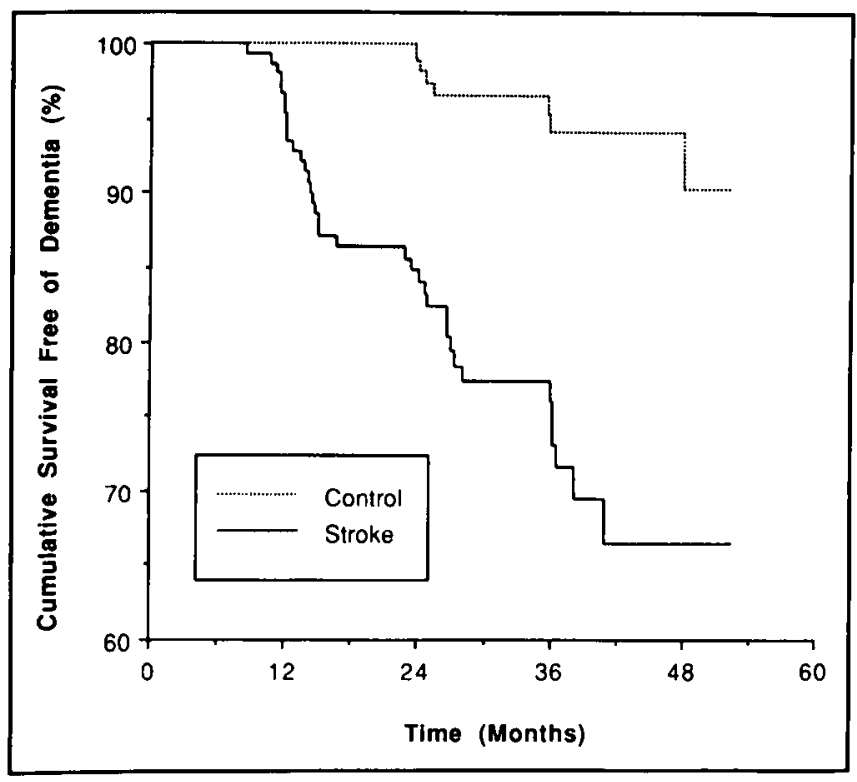

Figure. Kaplan-Meier analysis showing the cumulative proportion of subjects (stroke and control) surviving free of dementia during the follow-up period up to 52 months. Survival curves are significantly different by the log rank test $(\mathbf{p}<0.001)$.

years. The RR for the MMSE score was 0.77 (95\% CI, 0.70 to 0.84 ), indicating a protective effect with a higher score.

Discussion. Our study is the first, to our knowledge, to quantify the risk of dementia after ischemic stroke by providing an estimate of its incidence in a prospectively examined cohort of hospitalized patients aged $\geq 60$ years with ischemic stroke compared with a reference group of strokefree subjects. The observed incidence of 8.4 per 100 person-years is an extraordinarily high rate, far exceeding the IRs estimated by Kurtzke ${ }^{17}$ for many common neurologic disorders. This rate is considerably higher than the incidence of dementia (chiefly AD) found in community samples, ${ }^{18}$ including our own control group. Even when adjusted for demographic factors and baseline cognitive function, the risk of dementia associated with stroke was significantly elevated.

The validity of our risk estimates could be challenged because of potential biases related to selection of our controls, uncertainties regarding our analytic assumptions for outcome events, and diagnostic problems specific to incidence studies of dementia. First, the selection of our control cohort, composed of age-matched community volunteers, was guided by the need to assemble a reference group at risk for dementia comparable with the stroke group, except for the risk associated with the exposure of interest (ie, CVD). This sample could have been biased because community-residing volunteers tend to be healthier; thus, our control rates might be an underenumeration. However, we adjusted for demographic differences in our multivariate Cox model. Most importantly, the
Table 2. Relative risk of dementia based on Cox proportional-hazards model for stroke compared with control subjects adjusting for demographic factors in the groups overall (model $A)$ and in the community-based subgroups from northern Manhattan (model B)

\begin{tabular}{|lcc|}
\hline & \multicolumn{2}{c|}{ Relative risk (95\% CI) } \\
\cline { 2 - 3 } Variable & $\begin{array}{c}\text { Model A } \\
(\mathbf{n = 4 2 6})\end{array}$ & $\begin{array}{c}\text { Model B } \\
(\mathbf{n}=180)\end{array}$ \\
& & \\
Stroke vs control & $5.47(2.50-11.06)$ & $7.21(2.56-20.31)$ \\
Age (reference, 60-69 yr) & & \\
$\quad 70-79 \mathrm{yr}$ & $2.27(1.11-4.64)$ & $4.50(1.50-13.50)$ \\
$\quad 80+\mathrm{yr}$ & $5.73(2.59-12.68)$ & $4.96(1.28-19.16)$ \\
Education (reference, $13+\mathrm{yr})$ & & \\
$\leq 8 \mathrm{yr}$ & $3.00(1.21-7.42)$ & $4.38(0.86-22.25)$ \\
$9-12$ yr & $1.62(0.66-3.96)$ & $5.24(1.13-24.37)$ \\
Sex (men vs women) & $1.11(0.60-2.08)$ & $1.48(0.61-3.60)$ \\
Race (nonwhite vs white) & $1.79(0.87-3.66)$ & $1.46(0.57-3.69)$ \\
\hline
\end{tabular}

results of the second analysis confined to a population-based sample (model B) were similar. These considerations mitigate the concern about significant bias related to our control group selection.

A separate issue concerns the potential bias related to the possibility of a differential nonresponse rate from missing examinations due to refusals or mortality. It is difficult, if not impossible, to estimate this potential effect. In an informal analysis, we attempted to characterize those with "complete" compared with "incomplete" follow-up in both subject groups and found that there were only a few differences in their baseline features, supporting the view that the effect of missing examinations was probably nondifferential with respect to dementia as an outcome. A related analytic issue is the assumption by the Cox model that failure time (ie, incidence of dementia) was independent of a censoring event (eg, death). If the occurrence of dementia and mortality were related, the Cox model estimates of RR could be biased; however, that bias would be towards the null, since deaths were disproportionately higher in the stroke group compared with controls. Since we cannot be certain that subjects were nondemented at the time of censoring, our RRs should be regarded as an underestimate.

A third potential problem pertains to our method of diagnosing incident dementia. We chose the more rigorous approach of repeatedly examining a stroke sample at risk for dementia with standard clinical assessments to identify incident cases, rather than using the usual method of conducting prevalence surveys at different points in time. However, because we did not require a specific degree of cognitive decline to define incident disease, it could be argued that misclassification might have occurred regarding "prevalent" versus "incident" dementia, especially for subjects initially on the borderline. Since we used the same diagnostic methods for both groups, any potential overestimation of the RR for stroke compared with controls 
would be minimal. Finally, even after adjusting for baseline cognitive impairment using the MMSE, he stroke risk was still significantly elevated.

The validity of our study is supported by its conistency with the few uncontrolled investigations of smaller numbers of patients from which crude but comparable estimates of dementia incidence can be derived. Only the investigation of Kotila et al ${ }^{19}$ used similar methods, although controls were not assessed. Over 4 years of follow-up using repeated examinations, three of 37 infarct patients in their study met criteria for dementia. The crude "incidence ratio" of about $2 \%$ annually in a young stroke group would be consistent with our data, extrapolating from the rates among our older subjects. In another study of 100 nondemented patients with first stroke followed for 3 years, Aronovich et al ${ }^{20}$ found new dementia in 24\% using Kaplan-Meier analysis. No controls were studied. Our own Kaplan-Meier point estimates for the stroke sample (figure) indicate a similar proportion at 3 years.

The incidence of dementia related to CVD will, of course, vary depending on the definition of the exposure (and therefore the selection of the study population at risk) as well as the definition of the outcome. In our study, we examined elderly patients with a well-defined ischemic stroke whose outcome was "dementia after stroke." There have been incidence studies of other populations at risk for dementia, differing in the degree of "exposure" to CVD. Even though the different studies are not strictly comparable, we believe that an examination of the IRs of dementia from these studies is informative and helps to place our findings in a broader context. In population-based community studies, the IR has ranged from 0.17 to 0.71 per 100 person-years for "arteriosclerotic psychosis"21 or "multi-infarct dementia (MID),"22,23 including "mixed dementia." ${ }^{23}$ In a sample of 88 elderly volunteers free of stroke but with significant vascular risk factors, Rogers et $\mathrm{al}^{24}$ reported an "incidence ratio" of $1.6 \%$ for MID. Among 434 community volunteers aged 75 to 85 years studied by Katzman et $\mathrm{al}^{25}$ (of whom $6.8 \%$ had prior stroke or transient ischemic attacks), the IR for MID or mixed dementia was approximately 0.95 per 100 person-years. All these rates fall far below the rate of 8.4 per 100 person-years observed in our stroke group. Although differences in the study populations may account for the variation in rates, these findings suggest that increasing "levels" of CVD are associated with a corresponding increase in the risk of dementia, however defined.

Our findings raise the question of the mechanism( $(s)$ involved in intellectual deterioration occurring 1 or more years after ischemic stroke. How does this "delayed" dementia occur? Clarifying this issue will require longer follow-up of our stroke cohort, examining further the risks and risk factors in that group alone. Our study design will permit an analysis of the risk factors for incident dementia among those with stroke. Clinical observations in the stroke cohort thus far suggest several possibilities, which we consider hypotheses for further study. Clinically obvious recurrent stroke accounted for about one-fifth of the incident cases. Repeated brain infarctions leading to dementia is consistent with MID as defined by Hachinski et al. ${ }^{26}$ Another subgroup, similar in proportion, was characterized by events or disorders that could cause global cerebral ischemia or hypoxia (eg, repeated seizures, pulmonary disease, or cardiac disease with syncope, congestive heart failure, and so on), especially in elderly subjects who might be vulnerable to hypoxia or hypotension. A form of vascular dementia has been termed "cardiogenic dementia" 27 or "hypoperfusion dementia," 28 invoking this mechanism. A third subgroup was composed of a small number of "borderline" patients who subsequently fulfilled criteria for dementia after a slight decline. The entity of "questionable" or "borderline" dementia needs further investigation (specifically, whether it is a stable state or a stage in the progression to a diseased state), but low intellectual function at baseline has been found to be a risk factor for incident $\mathrm{AD} .^{25}$ The independent effect of the MMSE score at baseline in our sample is consistent with this observation. For the remaining incident cases, amounting to one-third of the sample, no obvious precipitating factor or other explanation could be identified. This is the most intriguing group. Progressive but "silent" CVD or co-existing $\mathrm{AD}$ unmasked by a stroke event are potential explanations for the deterioration. ${ }^{29}$ Follow-up brain imaging may clarify the former possibility, whereas autopsy confirmation will likely be needed to assess the latter mechanism. Our results are at least consistent with the thesis that a stroke event may accelerate the cognitive consequences of aging, including the effects of $\mathrm{AD}$.

The entity of "post-apoplectic dementia," despite its rejection by one contemporary critic, ${ }^{30}$ has long been recognized in the pathology literature, discussed at the turn of this century by both Binswanger in $1894^{31}$ and Alzheimer ${ }^{32,33}$ as a form of vascular dementia distinct from "encephalitis subcorticalis chronica progressiva" 31 and "arteriosclerotic brain degeneration." ${ }^{33}$ Regarding postapoplectic dementia, Alzheimer ${ }^{32}$ specifically offered the opinion that "after an apoplexia-also in younger individuals and also in those apoplexies that did not occur in the cortex and white matteroften a slowly progressing dementia develops [authors' emphasis], which is strikingly similar to dementia senilis [the disorder yet to be discovered pathologically by Alzheimer]. The anatomical basis of this type of dementia is-according to my own observations-a cortical alteration not only in the areas affected by the apoplexia which are very similar to the ones in dementia senilis."

A century later, there is still uncertainty regarding mechanisms, but our study has taken a first step towards clarifying dementia after stroke by reintroducing this entity and defining its risk. 


\section{Acknowledgments}

The authors thank Ms. Francisca Berment and Leslie Hanzawa, $\mathrm{RN}$, who coordinated the follow-up efforts of the Stroke and Aging Research Project. Dr. Henning Mast provided translations from the original German texts of Binswanger and Alzheimer, and Dr. James Goldman reviewed the histopathology of patient 10. Drs. J.P. Mohr, Isak Prohovnik, and Dale Hesdorffer provided critical comments on the manuscript.

\section{References}

1. Bousser M-G. Les conceptions actuelles des démences artériopathiques. L'Éncephale 1977;1II:357-372.

2. Tatemichi TK, Desmond DW, Mayeux R, et al. Dementia after stroke: baseline frequency, risks, and clinical features in a hospitalized cohort. Neurology 1992;42:1185-1193.

3. Rothman KJ. Modern epidemiology. Boston: Little, Brown, 1986.

4. Mahoney FI, Barthel DW. Functional evaluation: Barthel index. Md State Med J 1965;14:61-65.

5. Blessed G, Tomlinson BE, Roth $\mathrm{M}$. The association between quantitative measures of dementia and of senile change in the cerebral grey matter of elderly subjects. Br J Psychiatry 1968;114:797-811.

6. Stern Y, Andrews H, Pittman J, et al. Diagnosis of dementia in a heterogeneous population: part I. Development of a neuropsychological paradigm and quantified correction for education. Arch Neurol 1992;49:453-460.

7. Tatemichi TK, Desmond DW, Stern Y, Paik M, Sano M, Bagiella E. Cognitive impairment after stroke: frequency, patterns, and relationship to functional abilities. J Neurol Neurosurg Psychiatry 1994;57:202-207.

8. Williams JBW. A structured interview guide for the Hamilton Depression Rating Scale. Arch Gen Psychiatry 1988;45: 742-747.

9. Folstein MF, Folstein SE, McHugh PR. Mini-mental state: a practical method for grading the cognitive state of patients for the clinician. J Psychiatr Res 1975;12:189-198.

10. American Psychiatric Association. Diagnostic and statistical manual of mental disorders, 3rd ed, revised. Washington, DC: American Psychiatric Association, 1987.

11. Tatemichi TK, Desmond DW, Paik M, et al. Clinical determinants of dementia related to stroke. Ann Neurol 1993;33: $568-575$.

12. Mayeux R, Pittman J, Andrews H, Denaro J, Palestroni A, Stern Y. Reliability and consistency in the diagnosis of Alzheimer's disease and related conditions in communitybased studies labstract|. Neurology 1993;42(suppl 2):A233.

13. Hughes CP, Berg L, Danziger WL, Cohen LA, Martin RL. A new clinical scale for the staging of dementia. $\mathrm{Br} \mathrm{J}$ Psychiatry $1982 ; 140: 566-572$.
14. Kaplan EL, Meier P. Nonparametric estimation from incomplete observations. J Am Stat Assoc 1958;53:457-481.

15. Cox DR. Regression models and life tables. J R Stat Soc B 1972;34:187-220.

16. Schlesselman JJ. Case-control studies: design, conduct, analysis. New York: Oxford University Press, 1982.

17. Kurtzke JF. The current neurologic burden of illness and injury in the United States. Neurology 1982;32:1207-1214.

18. Kokmen E, Chandra V, Schoenberg BS. Trends in incidence of dementing illness in Rochester, Minnesota, in three quinquennial periods, 1960-1974. Neurology 1988;38:975-980.

19. Kotila M, Waltimo O, Niemi M-L, Laaksonene R. Dementia after stroke. Eur Neurol 1986;25:134-140.

20. Aronovich BD, Treves TA, Bornstein NM, Korczyn AD. Dementia after first stroke: 3 -years survival analysis labstract]. Cerebrovasc Dis 1992;2:216.

21. Akesson HO. A population study of senile and arteriosclerotic psychoses. Hum Hered 1969;19:546-566.

22. Hagnell O, Franck A, Grasbeck A, et al. Vascular dementia in the Lundby Study. Neuropsychobiology 1992;26:43-49.

23. Schoenberg BS, Kokmen E, Okazaki H. Alzheimer's disease and other dementing illnesses in a defined United States population: incidence rates and clinical features. Ann Neurol 1987;22:724-729.

24. Rogers RL, Meyer JS, Mortel KF, Mahurin RK, Judd BW. Decreased cerebral blood flow precedes multi-infarct dementia, but follows senile dementia of Alzheimer type. Neurology 1986;36:1-6.

25. Katzman R, Aronson M, Fuld P, et al. Development of dementing illnesses in an 80-year-old volunteer cohort. Ann Neurol 1989;25:317-324.

26. Hachinski VC, Lassen NA, Marshall J. Multi-infarct dementia: a cause of mental deterioration in the elderly. Lancet 1974;2:207-210.

27. Cardiogenic dementia leditoriall. Lancet 1977;1:27-28.

28. Sulkava R, Erkinjuntti T. Vascular dementia due to cardiac arrhythmias and systemic hypotension. Acta Neurol Scand 1987;76:123-128.

29. Tatemichi TK. How acute brain failure becomes chronic: a view of the mechanisms of dementia related to stroke. Neurology $1990 ; 40 ; 1652-1659$.

30. Drachman DA. New criteria for the diagnosis of vascular dementia: do we know enough yet? Neurology $1993 ; 43: 243$ 245 .

31. Binswanger $O$. Die Abgrenzung der allgemeinen progressiven Paralyse. Berl Klin Wochenschr 1894;31:1103-1105; 1137-1139; 1180-1186.

32. Alzheimer A. Neuere Arbeiten uber die Dementia senilis und die auf atheromatoser Gefasserkrankung basierdenden Gehirnkrankheiten. Monatsschr Psychiatr Neurol 1898;3:101-115.

33. Alzheimer A. Die Seelenstorungen auf arteriosklerotischer Grundlage. Allg Z Psychiatr Psych Gerict Med 1902;59:695711. 